\title{
Amelioration of renal damage by administration of anti-thymocyte globulin to potential donors in a brain death rat model
}

\author{
F Cicora, ${ }^{\star \dagger}$ P. Stringa, ${ }^{\star \S}$ D. Guerrieri, \\ J. Roberti, ${ }^{\dagger}$ N. Ambrosi, ${ }^{9}$ F. Toniolo, ${ }^{\dagger}$ \\ P. Cicora, ${ }^{\dagger}$ G. Palti, ${ }^{\ddagger}$ D. Vásquez ${ }^{\dagger}$ and \\ C. Raimondi* \\ ${ }^{*}$ Transplant Program, Medicine Faculty, National \\ University of La Plata, La Plata, ${ }^{\dagger}$ Foundation for \\ Research and Assistance in Kidney Disease \\ (FINAER), ${ }^{*}$ Renal Transplant Unit, German \\ Hospital- Hospital Alemán, ${ }^{\circledR}$ Multiorgan \\ Transplant Institute, Favaloro Foundation, and \\ 'Pharmacology Department, Medicine Faculty, \\ University of Buenos Aires, Buenos Aires, \\ Argentina
}

Accepted for publication 8 May 2012

Correspondence: Mr J. Roberti, Austria 2381,

5D; 1425 Buenos Aires, Argentina.

E-mail: javierroberti@gmail.com

\begin{abstract}
Summary
Brain death (BD), a non-immunological factor of renal injury, triggers an inflammatory process causing pathological signs of cell death in the kidney, such as necrosis and apoptosis. Kidneys from brain dead donors show lower success rates than kidneys from living donors and one strategy to improve transplantation outcome is to precondition the donors. For the first time, anti-rat thymoglobulin (rATG) was administered in an experimental brain death animal model to evaluate if it could ameliorate histopathological damage and improve organ function. Animals were divided into three groups: $\mathrm{V}(n=5)$ ventilated for $2 \mathrm{~h}$; $\mathrm{BD}(n=5)$ brain death and ventilated for $2 \mathrm{~h}$; and BD+rATG $(n=5)$ brain death, ventilated for $2 \mathrm{~h}$, rATG was administered during brain death $(10 \mathrm{mg} / \mathrm{kg})$. We observed lower creatinine levels in treatment groups (means): $\mathrm{V}, 0.88 \pm 0.22 \mathrm{mg} / \mathrm{dl} ; \mathrm{BD}, 1.37 \pm 0.07 \mathrm{mg} / \mathrm{dl}$; and BD+rATG, $0.64 \pm 0.02 \mathrm{mg} / \mathrm{dl}$ (BD versus $\mathrm{BD}+\mathrm{rATG}, P<0.001$ ). In the BD group there appeared to be a marked increase of ATN, whereas ATN was decreased significantly in the rATG group $(\mathrm{V}, 2.25 \pm 0.5$ versus $\mathrm{BD}$, $4.75 \pm 0.5, P<0.01$; BD+rATG, $2.75 \pm 0.5$ versus $\mathrm{BD} 4.75 \pm 0.5 P<0.01$ ). Gene expression was evaluated with reverse transcription-polymerase chain reaction; tumour necrosis factor (TNF)- $\alpha$, interleukin (IL)-6, C3, CD86 showed no significant difference between groups. Increased IL-10 and decreased CCL2 in BD+rATG compared to BD (both cases $P<0 \cdot 01$ ). Myeloperoxidase was increased significantly after the brain death setting (V: $32 \pm 7 \cdot 5$ versus BD: $129 \pm 18$ ). Findings suggest that $\mathrm{rATG}$ administered to potential donors may ameliorate renal damage caused by BD. These findings could contribute in the search for specific cytoprotective interventions to improve the quality and viability of transplanted organs.
\end{abstract}

Keywords: acute tubular necrosis, brain death, IL-10, MCP-1, thymoglobulin

\section{Introduction}

Critical care teams work to avoid or minimize injury before brain death. However, once brain death is diagnosed and the patient or their family express their wish to donate organs, efforts are directed to improve organ quality and the subsequent transplant outcome [1]. Brain dead donors represent the most important source of kidneys for transplant [2], but transplanted kidneys from these donors suffer from lower success rates than grafts from living donors [3]. These poor results may be attributed to the pathophysiological changes that take place during brain death [3]. Different approaches have been used to improve transplant outcomes, including treating the recipient, intervening with a preservation solution or preconditioning the donor [2]. In an elegant review of donor intervention and organ preservation, Feng [4] explained that donor management has the best potential to improve the quality and size of the organ supply.

Brain death causes an increase in parasympathetic tone and, subsequently, intense sympathetic stimulation with endogenous catecholamine release. This process results in vasoconstriction, secondary tissue ischaemia, changes in adenosine triphosphate (ATP production) and the production of oxygen-free radicals. Additionally, the production of cytosolic calcium increases and nitric oxide synthase concentration is augmented. The subsequent hypotensive stage, which is characterized by diminished sympathetic activity, may reduce oxygen availability to the tissues [5]. Brain death 
is also associated with higher cytokine levels and systemic proinflammatory changes [6]. Immunological responses activate endothelial cells and proinflammatory genes [7], and the inflammatory process generates pathological signs of cell death such as kidney necrosis and apoptosis. Moreover, activation of adhesion molecules and inflammatory mediators leads to a non-specific immune response in the kidney that may cause acute rejection in the immediate posttransplantation period [8].

Acute tubular necrosis (ATN) in kidneys from brain dead donors is generally treated with interventions directed at recipients. However, this approach requires an important immunosuppressive load, and consequent higher costs for the health-care system are probable. As brain death has a specific cytokine profile, brain dead donors require a unique approach to preserve their organs [9]. Donors pretreated with steroids show decreased proinflammatory cytokine expression in tissues and serum [10]. In an animal model, Pratschke et al. [7] observed that preconditioning brain dead donors with steroids reduces ischaemia/reperfusion injury rate in kidney recipients.

Anti-thymocyte immunoglobulin (Ig) (ATG) (Thymoglobulin ${ }^{\circledR}$; Genzyme, Cambridge, MA, USA) is a purified fraction of IgG from the sera of rabbits immunized against human thymocytes and is administered commonly to recipients in renal transplant settings. In a previous in-vitro study, ATG antibodies affected the binding and/or expression of ligands such as intercellular adhesion molecule 1 (ICAM-1) and surface molecules such as lymphocyte functionassociated antigen 1 (LFA1), which intervene in the leucocyte-endothelium interaction. ATG also contains antiCCR7, anti-CXCR4 and anti-CCR5 antibodies that inhibit leucocyte homing and trafficking to the graft by means of binding to chemokine receptors [11].

No strategy directed at brain dead potential donors has been demonstrated to be sufficiently reliable and consistent. To the best of our knowledge, this is the first experimental study in which ATG was administered to brain dead potential donors. We explored whether administering ATG to the donor organ-to-be diminished histological damage and improved renal function in the organ to be transplanted. Of note, thymoglobulin was not associated with immunosuppressants or other drugs, as is generally the case in clinical contexts to evaluate thymoglobulin without the complicating effects of other drugs.

\section{Materials and methods}

\section{Animals}

Fifteen Sprague-Dawley male rats $(300 \pm 30$ g; Veterinary Faculty, University of Buenos Aires, Argentina) were submitted to controlled macro- and microenvironmental conditions, with access to water and standard laboratory chow ad libitum. All experiments were performed in accordance with the guidelines set by the National Institutes of Health (NIH publication no. 28, revised 1996).

\section{Generation and characterization of rATG}

The rabbit anti-rat thymocyte immunoglobulin (rATG) was provided by Genzyme and produced analogously to commercial ATG (Thymoglobulin ${ }^{\circledR}$ ). Rabbits were immunized with a mixture of thymocytes of four strains of rats (Sprague-Dawley, F344 Fisher, Lewis and Long Evans). Thymocyte suspensions were prepared with thymi from various donor rats. Fifty New Zealand White rabbits were immunized twice, 2 weeks apart, and terminally bled 2 weeks after the last immunization. Total rabbit IgG obtained from the serum was pooled and purified with a process similar to Thymoglobulin ${ }^{\circledR}$ (Genzyme). Control rabbit IgG was purified similarly from whole normal rabbit serum.

\section{Experimental design}

Rats were divided randomly into three groups: group $\mathrm{V}$ $(n=5)$ animals without brain death that were ventilated mechanically for $2 \mathrm{~h}$; group $\mathrm{BD}(n=5)$ animals with brain death that were ventilated mechanically for $2 \mathrm{~h}$; and group $\mathrm{BD}$ and rATG $(n=5)$ animals with brain death that were ventilated mechanically for $2 \mathrm{~h}$. Immediately after the brain death diagnosis, rATG was administered intravenously (10 mg/kg; Genzyme). The dose was suggested by the manufacturer.

All rats were anaesthetized with a combination of ketamine (80 mg/kg; Hollyday, Buenos Aires, Argentina) and midazolam (5 mg/kg; Richmond, Buenos Aires, Argentina) administered intraperitoneally, and lidocaine was used as a local anaesthetic. Animals were placed in a sternal recumbent position and a $0 \cdot 5-\mathrm{cm}$-long front lateral trepanation was performed to insert a Fogarty no. 3 balloon catheter. Animals were placed in the dorsal decubitus position to place a no. 22 cannula in the right carotid artery and another no. 22 cannula in the left jugular vein. The artery pathway was connected to a multi-parametric DYNE MCO-300-07 monitor to control blood pressure. The venous pathway was connected to an infusion pump to administer physiological solution $(5 \mathrm{ml} / \mathrm{kg} / \mathrm{h})$. Animals were ventilated with a Neo Net ventilator (Tecme SA, Cordoba, Argentina) (VT, 3 ml; TI, 0.25; I : E, 1:2.0; FR, 80; Peep, 5; FIO, 40\%). The balloon was then inflated at a speed of $50 \mu \mathrm{l} / \mathrm{min}$ to reach $600-800 \mu \mathrm{l}$ and produce brain death. Brain death was diagnosed by the absence of a corneal reflex and a positive apnoea test. Animals were ventilated for $2 \mathrm{~h}$, and blood pressure was kept constant at 60-120 mmHg (Fig. 1). Noradrenaline was administered $(20 \mu \mathrm{g} / \mathrm{ml}$; Biol, Buenos Aires, Argentina) to stabilize hypotensive $(<60 \mathrm{mmHg})$ animals within the desired blood pressure range. After $2 \mathrm{~h}$ of brain death [12], a blood sample was collected, the animals were killed and a left kidney sample was collected for histopathological analysis. 


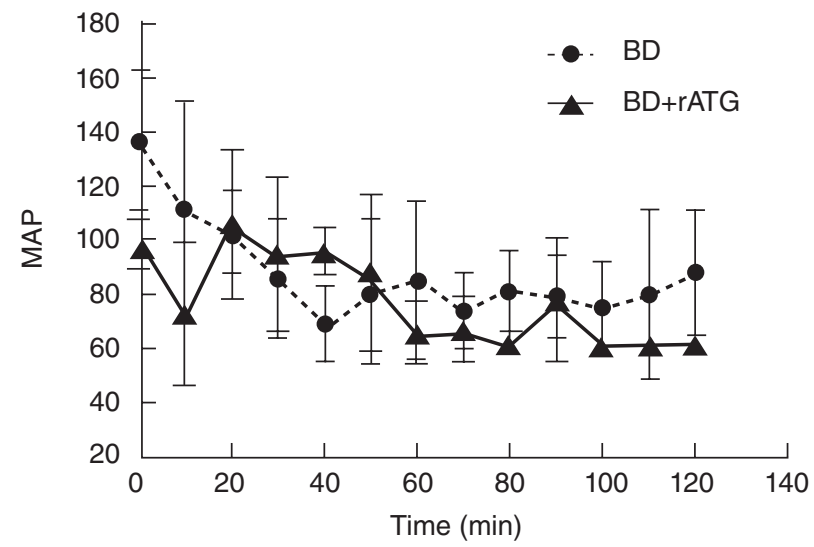

Fig. 1. Mean blood pressure evolution. Shown are changes in mean blood pressure during brain death induction in rats. After brain death was diagnosed, animals were ventilated for $2 \mathrm{~h}$; during that time, blood pressure was kept constant within 60-120 mmHg. In the cases of hypotension, animals were treated with noradrenaline. Similar levels were kept for the ventilated group (data not shown).

\section{Blood analysis}

Blood samples were used to determine urea and creatinine levels, as quantified by kinetic ultraviolet and kinetic colorimetric assays, respectively (Mindray 300).

\section{Renal histopathology}

Pathological samples were analysed by a pathologist blind to the group assignments. The kidneys were fixed in a $10 \%$ neutral buffered formalin solution, embedded in paraffin and used for histopathological examination. Sections $(4 \mu \mathrm{m}$ thick) were cut, deparaffinized, hydrated and stained with haematoxylin and eosin. The renal sections were examined for cortical tubular epithelial necrosis in a blind fashion. Counts were performed in 10 fields of $1 \mathrm{~mm}^{2}$ and assigned for necrosis severity using scores on a scale from 1-5: 1 (<5\%), 2 (5-25\%), 3 (25-50\%), $4(50-75 \%)$ and $5(>75 \%)$ [13].

\section{Quantitative polymerase chain reaction ( $\mathrm{PPCR}$ )}

Intrarenal mRNA levels were analysed to determine the expression of tumour necrosis factor (TNF)- $\alpha$, interleukin (IL)-6, IL-10, CCL2, C3 and CD86 in the experimental groups. Samples were stored at $-80^{\circ} \mathrm{C}$ in RNALater. Total RNA was purified from $30 \mathrm{mg}$ of tissue with an RNeasy kit (Qiagen, Courtabouef, France). RNA integrity and quantity were evaluated by spectrophotometry at 260 and $280 \mathrm{~nm}$. Complementary DNA (cDNA) was obtained with reverse transcriptase using a First Strands Kit (SABiosciences, Qiagen) from $0 \cdot 7 \mu \mathrm{g}$ of total isolated RNA. qPCR analyses were performed with in an ABI Prism 7500 instrument and a SYBR Green SuperScript kit (Qiagen). The glyceraldehyde3-phosphate dehydrogenase (GAPDH) gene was used as a control and its expression levels were used to normalize the data. GAPDH levels were similar in all groups (data not shown). To determine expression level, serial dilutions of calibrator cDNA were made and a standard curve for each gene was created. Ct values were compared to the cDNA concentrations. The curve was used to determine the differences in the Ct value in each sample. The concentration of each sample was calculated as a percentage of mRNA relative to the calibrator, as per the $\Delta \Delta \mathrm{CT}(2-\Delta \Delta \mathrm{CT})$ method. Gene expression results are reported as the variation or change relative to the ventilated group; the expression value for each gene was fixed at 1 .

\section{Renal myeloperoxidase}

Renal myeloperoxidase (MPO) activity, which indicates polymorphonuclear leucocyte (PMN) infiltration into the kidney, was measured according to the protocol of Singbartl et al. $[14,15]$. First, samples were homogenized in ice-cold $20 \mathrm{mM} \mathrm{KPO}_{4}$ buffer. After removing the supernatants by centrifugation at $17000 \mathrm{~g}$, the pellets were resuspended in ice-cold $20 \mathrm{mM} \mathrm{KPO}_{4}$ buffer followed by two more centrifugations. Then, $0 \cdot 5 \%(\mathrm{w} / \mathrm{v})$ hexacyltrimethylammonium bromide- $10 \mathrm{mM}$ ethylenediamine tetraacetic acid (EDTA) in $50 \mathrm{mM} \mathrm{KPO}_{4}$ was added to the pellet. The suspensions were sonicated, freeze-thawed and incubated for $20 \mathrm{~min}$ at $4^{\circ} \mathrm{C}$. Supernatants $(17000 \mathrm{~g}$ ) were used to measure MPO with 3,3'-5,5' tetramethylbenzidine (TMB) (1:5 v/v), and the absorbance was recorded at $460 \mathrm{~nm}$. Results are expressed as units of MPO/mg supernatant protein as determined by the bicinchoninic acid assay (Pierce Chemical Co., Rockford, IL, USA).

\section{Statistical analysis}

Data are expressed as mean \pm standard deviation (s.d.). Differences between groups or conditions were analysed by analysis of variance (ANOVA). When ANOVA was significant, multiple comparisons were performed with post-hoc Student-Newman-Keuls test to locate the source of difference. Non-parametric variables were analysed with the Krustal-Wallis non-parametric test of ANOvA. A $P$-value of $<0.05$ was considered statistically significant.

\section{Results}

\section{Plasma urea and creatinine concentrations}

Plasma levels of urea and creatinine were quantified in all groups $2 \mathrm{~h}$ after the brain death diagnosis. Urea levels in the groups were as follows: ventilated group (V), $0.43 \pm 0.014 \mathrm{mg} / \mathrm{dl}$ and brain death group (BD), $0 \cdot 57 \pm 0 \cdot 1 \mathrm{mg} / \mathrm{dl}$. The difference between these two 
Fig. 2. Urea and creatinine scores. Two h after brain death (BD) was diagnosed, blood samples were collected and urea (a) and creatinine (b) were evaluated. Data are expressed as mean \pm standard deviation (s.d.), $n=5$. ${ }^{* *} P<0 \cdot 01$ and ${ }^{* * *} P<0 \cdot 001$ for comparison with the BD group.
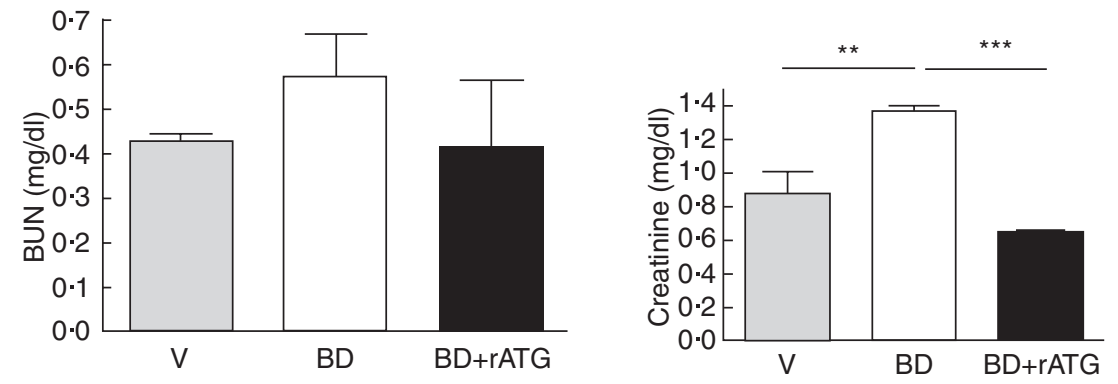

groups was not statistically significant. Mean urea was $0.41 \pm 0.15 \mathrm{mg} / \mathrm{dl}$ in the rATG-treated group (BD and rATG). The difference between this group and the $\mathrm{BD}$ group was not significant. Mean creatinine levels were $0 \cdot 88 \pm 0.22 \mathrm{mg} / \mathrm{dl}$ in the $\mathrm{V}$ group; $1.37 \pm 0.07 \mathrm{mg} / \mathrm{dl}$ in the BD group (V versus BD: $P<0.01$ ); and $0.64 \pm 0.02 \mathrm{mg} / \mathrm{dl}$ in the $\mathrm{BD}$ and $\mathrm{rATG}$ group. The difference in plasma creatinine between the $\mathrm{BD}$ and rATG group and the BD group was significant $(P<0 \cdot 001)$ (Fig. 2$)$.

\section{Necrosis and MPO}

Kidney necrosis was evaluated with haematoxylin and eosin staining of renal tissue. The BD group showed a significantly higher ATN score than that in the $\mathrm{V}$ group at $2 \mathrm{~h}$ after diagnosis (BD: $4.75 \pm 0.5$ versus $\mathrm{V}: 2 \cdot 25 \pm 0 \cdot 5, P<0 \cdot 01$; Fig. 3a). The ATN score decreased to $2 \cdot 75 \pm 0 \cdot 5$ in the thymoglobulin-treated group, which was a significant difference compared to that in the BD group (BD and rATG: $2 \cdot 75 \pm 0.5$ versus $\mathrm{BD}, 4.75 \pm 0.5, P<0 \cdot 01)$. Figure $3 \mathrm{~b}$ shows representative kidney tissue sections of each experimental group. Renal MPO increased significantly after brain death (V: $32 \pm 7.5$ versus BD: $129 \pm 18$, Fig. $3 \mathrm{c}$ ) indicating an almost fourfold increase in tissue PMN content. This increase in MPO levels decreased significantly in the BD and rATG group (BD: $129 \pm 18$ versus BD and rATG: $110 \pm 14$, $P<0 \cdot 05)$.

\section{Quantification of inflammatory mediators}

Expression levels of TNF- $\alpha$, IL-6, IL-10, monocyte chemotactic protein 1 (CCL2), C3 and CD86 were evaluated in all experimental groups using real-time PCR (Fig. 4 and Table 1 for RT-PCR primers). Inflammation was assessed through expression of the proinflammatory genes TNF- $\alpha$ and IL-6. In the BD group, TNF- $\alpha$ was 20 times higher than that in the

Fig. 3. Acute tubular necrosis (ATN) quantification. (a) ATN score in the three experimental groups. Tables show mean \pm standard deviation values (s.d.) of all animals in the three groups. ${ }^{* *} P<0.005$ compared to the brain death group (BD). (b) Haematoxylin and eosin (H\&E) staining, original magnification $\times 20$. Ventilated (V) group: ATN $20 \%$ of parenchyma. Flattened focal tubular epithelium and variable dilatation observed. BD group: ATN 70\% of parenchyma. Denudation of epithelial membrane and haemorrhage focus. BD+rATG group: ATN 30\% of parenchyma. Expanded tubules with intraluminal cell casts and loss of epithelial membrane or replacement by squamous epithelium. (c) Renal polymorphonuclear (PMN) infiltration was analysed with myeloperoxidase (MPO) activity determinations. Results are expressed as units of $\mathrm{MPO} / \mathrm{mg}$ of protein. Data are expressed as mean \pm standard deviation (s.d.), $n=5$. ${ }^{\star} P<0.05$ and for comparison with BD group versus $\mathrm{BD}+\mathrm{rATG}$.

(a)
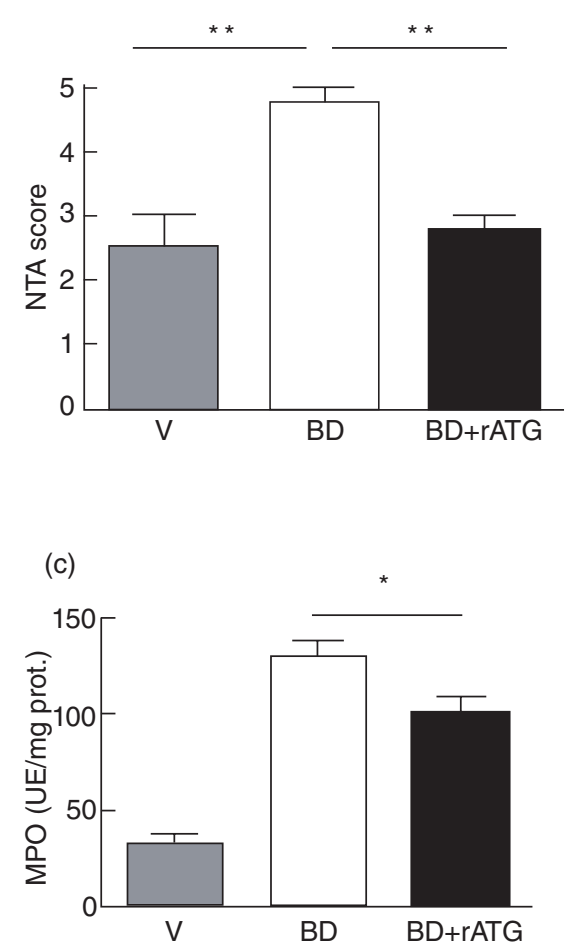

(b)

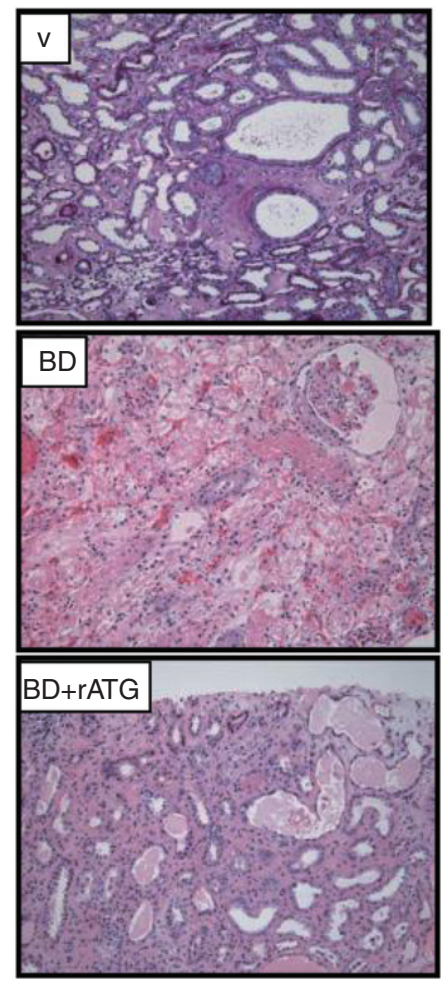


TNF- $\alpha$
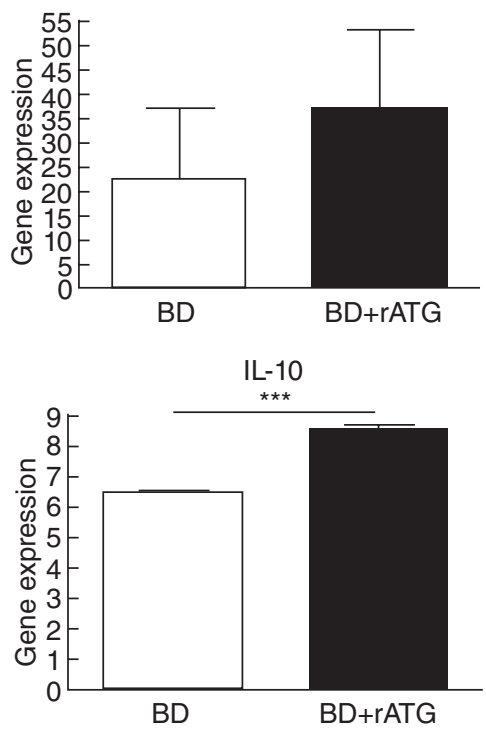

CD86

Fig. 4. Determination of inflammatory mediators. Intrarenal levels of mRNA for tumour necrosis factor-alpha, interleukin (IL)-6, IL-10, monocyte chemotactic protein 1 (MCP-1), C3 and CD86 in kidneys from brain death group $(\mathrm{BD})$ and treatment group (BD+rATG). ${ }^{\star} P<0.05$ and ${ }^{* * *} P<0.001$ comparing $\mathrm{BD}$ versus $\mathrm{BD}+\mathrm{rATG}$.

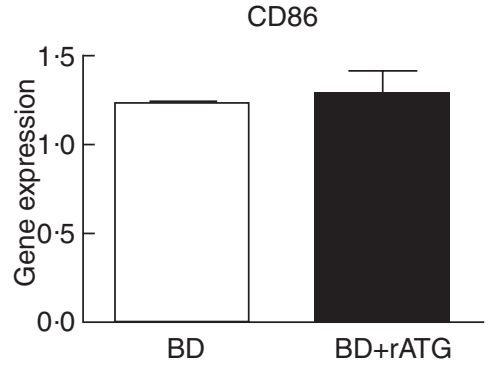

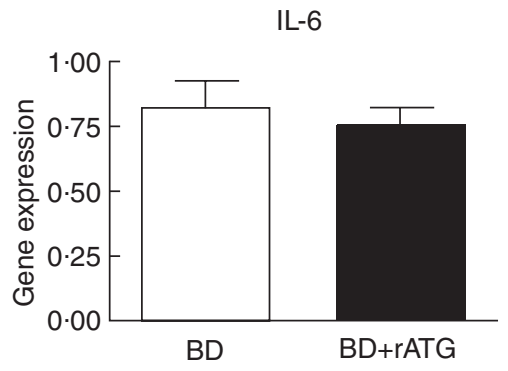

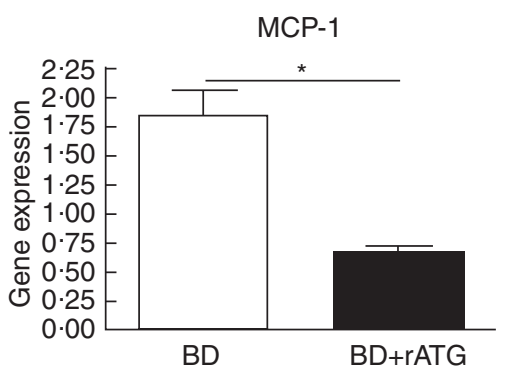

C3

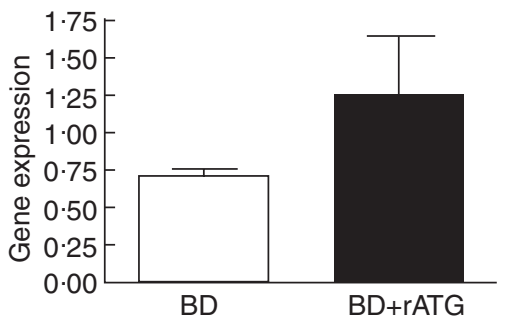

V group. Changes in TNF- $\alpha$ and IL- 6 between the BD and $\mathrm{BD}$ and rATG groups were not significant $(P>0 \cdot 05)$. However, animals in the BD and rATG group showed a statistically significant increase in IL-10 compared to that in the BD group $(P<0 \cdot 001)$.

We evaluated leucocyte migration and antigen presenter cell activation through CCL2 and CD86 expression. CCL2

Table 1. Gene-specific reverse transcription-polymerase chain reaction primers.

\begin{tabular}{lll}
\hline Gene & & \multicolumn{1}{c}{ Primer sequence } \\
\hline TNF-alpha & $5^{\prime}$ & ACCAGCCAGGAGGGAGAACA \\
IL-6 & $3^{\prime}$ & CGGATCATGCTTTCCGTGCTC \\
& $5^{\prime}$ & CATTCTGTCTCGAGCCCACC \\
IL-10 & $3^{\prime}$ & AGTGGTATATACTGGTCTG \\
& $5^{\prime}$ & TACTGGCTGGAGTGAAGACC \\
MCP-1 & $3^{\prime}$ & CAAGTAACCCTTAAAGTCCTG \\
& $5^{\prime}$ & AGACAGAGGCCAGCCCAGAAA \\
C3 & $3^{\prime}$ & TTGAGCTTGGTGACAAATACT \\
& $5^{\prime}$ & ACTAGTGCTACTGCTGCTGT \\
CD86 & $3^{\prime}$ & TGACAGTGACTGGGACATC \\
& $5^{\prime}$ & GGGAATCCTTTTCTCGGTGT \\
& $3^{\prime}$ & AGCCTGGTTATCCCTATCAAA \\
\hline
\end{tabular}

IL: interleukin; MCP-1: monocyte chemotactic protein 1; TNF: tumour necrosis factor. expression was 1.9 times higher in the BD group compared to that in the $\mathrm{V}$ group. CCL2 expression decreased significantly in the rATG group $(P<0 \cdot 05)$. The difference in CD86 between the groups was not statistically significant. Complement C3 in the kidneys was evaluated as a determinant of innate immune activation level, but the difference between the groups was not significant.

\section{Discussion}

The significant pathophysiological alterations caused by brain death in a potential organ to be transplanted result in inflammation and injury, which eventually affect posttransplant function and graft survival [16]. A comprehensive understanding of this process is necessary to improve intervention strategies. The results of our study suggest that rATG administered to donors could improve kidneys affected by brain death. Specifically, the treatment group showed a significant decrease in ATN score and creatinine values, a significant increase in IL-10 expression and a significantly lower in-situ expression of CCL2.

The precise mechanism underlying the immunosuppressive efficacy of rATG in renal transplant recipients is unclear, although it has been attributed primarily to $\mathrm{T}$ cell depletion. However, several other mechanisms may also be responsible. 
According to data mainly, but not exclusively, from preclinical studies, rATG modulates the expression of several lymphocyte surface antigens involved in a wide variety of functions ranging from $\mathrm{T}$ cell activation to endothelial adherence. rATG also activates certain transcription factors and interferes with numerous immune cell processes such as cytokine production, chemotaxis, endocytosis, stimulation and proliferation $[11,17,18]$.

Renal injury is generally assessed using functional markers such as creatinine, and a histological damage marker such as ATN. These markers decreased in our treatment group, which may indicate that rATG administered after brain death ameliorated renal damage caused by brain death. MPO, an enzyme found in neutrophils and monocyte phagosomes, is used as an indirect indicator of neutrophil infiltration. The lower levels of MPO in the treatment group may have occurred because rATG depletes neutrophils and interferes with leucocyte-endothelium interactions through downregulation of integrin CD11a/CD18 (LFA-1) surface expression $[17,18]$. The depletion of neutrophils in kidney tissue may be an indirect effect of rATG rather than a direct effect of antibody depletion. It is possible that some antigens detected by the antibody are common to thymocytes and neutrophils. However, another explanation may be that circulating $\mathrm{T}$ cells are depleted, and that these are responsible for recruiting neutrophils.

During brain death, renal tissue is damaged through an inflammatory process involving endothelial injury, leucocyte infiltration and tubular epithelial cell damage [9]. During this process, there appears to be increased in-situ and systemic expression of proinflammatory cytokines, such as TNF- $\alpha$, IL-1, IL-6 and IL-8, leucocyte activation and ICAM-1 and vascular adhesion molecule (VCAM-1) expression $[19,20]$. Surprisingly, we did not find any difference in the in-situ expression of TNF- $\alpha$, IL- 6 or C3 between the groups. This may be explained by the augmented expression of TNF- $\alpha$ and IL- 6 within the first $3 \mathrm{~h}$ after rATG administration in addition to their increase as a result of brain death, as shown by Guttman et al. [21].

Complement $\mathrm{C} 3$ gene expression may not be related to the amount of C3 activated, which is relevant to kidney damage. The importance of local renal complement C3 synthesis in the pathogenesis of kidney damage after transplantation has been described previously [16]. Donor kidneys from C3 knock-out mice show better survival rates after transplantation than kidneys from wild-type mice. Even though induction of renal complement C3 gene expression has been credited to ischaemia/reperfusion injury during transplantation, the importance of donor C3 has been observed [22]. Induction of complement components in kidneys from deceased donors occurs after cold ischaemia [23]. However, our results did not demonstrate that donor local renal complement expression in grafts from brain dead donors is important for a good outcome after transplantation. More studies are necessary to determine if local renal complement expression and activation in brain dead donors might actually be responsible for transplant outcome.

Although proinflammatory mediator expression between the groups was not significantly different, decreased ATN in the rATG group may be explained by significantly increased in-situ over-expression of IL-10, which inhibits proinflammatory cytokines such as TNF- $\alpha$. IL-10 is a pleiotropic, antiinflammatory cytokine with immunosuppressive properties and the main inhibitory cytokine of TNF- $\alpha$. Zeller et al. [24] demonstrated that IL-10 and transforming growth factor (TGF)- $\alpha$ have an additive effect as a tolerance inducer. IL-10 is associated with a diminished probability of interstitial fibrosis and renal tubular atrophy when it is over-expressed in situ in the renal parenchyma. Regulatory T cells seem to be resistant to ATG-mediated $\mathrm{T}$ cell depletion and can even suppress the recovery of $\mathrm{T}$ cells with an effector phenotype [25]. However, Lopez et al. [25] showed that low, nondepleting doses of rATG to peripheral blood mononuclear cells could expand human $\mathrm{CD} 4^{+} \mathrm{CD} 25^{+}$forkhead box protein $3\left(\right.$ FoxP $\left.^{+}\right)$regulatory $\mathrm{T}$ cells with suppressive properties in vitro. The potential source of IL-10 was not investigated in this study, but ATG can generate changes in different lymphocyte cell subsets in blood and regulatory $\mathrm{T}$ cells expansion [26]. Liu et al. [27] showed that when CD4 ${ }^{+}$cells are pretreated with ATG the regulatory $\mathrm{T}$ cells are expanded, inducing IL-10 secretion. Of note, thymoglobulin has different effects on each lymphocyte subset. We cannot verify this finding, because immunophenotypic characterization of cell infiltrates was not performed in our study. ATG-mediated regulatory $\mathrm{T}$ cell expansion is a result of ATG-mediated reprogramming of $\mathrm{CD}^{+}$and is dependent on signal transducer and activator of transcription-3 (STAT3). These modified $\mathrm{CD} 4^{+} \mathrm{T}$ cells induce IL-10 secretion only in the presence of intact STAT3 signalling. IL-10 secretion subsequently promotes $\mathrm{CD}_{14}{ }^{+}$and $\mathrm{CD} 11^{+}$tolerogenic dentritic cells [28].

Another result worthy of note was the diminished expression of CCL2 in renal tissue of the treatment group. De Vries et al. [9] demonstrated that CCL2 increases significantly in grafts, attracting macrophages during brain death. CCL2, a monocyte chemoattractant protein from the $\mathrm{C}$ - $\mathrm{C}$ chemokine family, is associated with various diseases and inflammatory processes [13]. It binds to its G-protein-coupled receptor and triggers monocyte/macrophage migration and infiltration. It also stimulates an inflammatory response that increases IL-6 and interferon (IFN)- $\gamma$ [29]. Decreased CCL2 levels in the rATG-treated group may have caused lower levels of monocyte/macrophage infiltration in renal tissue and lower production of proinflammatory mediators, which are responsible for ATN of renal tubular epithelial cells. It is not yet clear how ATG diminishes CCL2, but a possible explanation may be the presence of anti-CCL2 antibodies in rATG.

rATG is administered typically to renal transplant recipients, and it has begun to play a role in preventing ischaemia/ reperfusion injury. Aiello et al. [11] demonstrated that rATG 
is effective in preventing impaired renal function and tissue damage when the drug is administered to recipients at $22 \mathrm{mg} / \mathrm{kg}$ beginning $2 \mathrm{~h}$ before transplantation.

This is the first experimental study to administer ATG to potential donors during brain death. Our results suggest that ATG administered during brain death may ameliorate renal injury before cold ischaemia. The main limitation of this study is the observation time of $2 \mathrm{~h}$; therefore, it would be beneficial to design future experimental models with longer observation times. Additionally, while the RT-PCR data showed changes in gene expression, these changes are not always related directly to protein expression. Some cytokines can be controlled post-translationally; therefore, mRNA levels may not be proportional to protein levels. Further research should include transplantation in a model and rATG administration to donors and recipients to compare effects. In conclusion, considering that research on donor management and organ preservation offers realistic hopes to improve transplantation outcomes [4], our findings could contribute in the search for specific cytoprotective interventions to improve the quality and viability of transplanted organs.

\section{Acknowledgements}

This work was financed by Foundation for Research and Assistance in Kidney Disease (FINAER), Buenos Aires and Genzyme de Argentina S.A.

\section{Disclosure}

The authors declare no conflicting interests.

\section{References}

1 Nijboer WN, Ottens PJ, van Dijk A, van Goor H, Ploeg RJ, Leuvenink HG. Donor pretreatment with carbamylated erythropoietin in a brain death model reduces inflammation more effectively than erythropoietin while preserving renal function. Crit Care Med 2010; 38:1155-61.

2 Diethelm AG, Blackstone EH, Naftel DC et al. Important risk factors of allograft survival in cadaveric renal transplantation. A study of 426 patients. Ann Surg 1988; 207:538-48.

3 Schuurs TA, Gerbens F, van der Hoeven JA et al. Distinct transcriptional changes in donor kidneys upon brain death induction in rats: insights in the processes of brain death. Am J Transplant 2004; 4:1972-81.

4 Feng S. Donor intervention and organ preservation: where is the science and what are the obstacles? Am J Transplant 2010; 10:1155-62.

5 Demetrios J, Kutsogiannis G, Pagliarello G et al. Medical management to optimize donor organ potential: review of the literature. Can J Anesth 2006; 53:820-30.

6 Morariu AM, Schuurs TA, Leuvenink HG et al. Early events in kidney donation: progression of endothelial activation, oxidative stress and tubular injury after brain death. Am J Transplant 2008; 8:933-41.
7 Pratschke J, Kofla G, Wilhelm MJ et al. Improvements in early behavior of rat kidney allografts after treatment of the brain dead donor. Ann Surg 2001; 234:732-40.

8 Pratschke J, Wilhelm MJ, Kusaka M et al. Accelerated rejection of renal allografts from brain dead donors. Ann Surg 2000; 232:26371.

9 De Vries DK, Lindeman JHN, Ringers J, Reinder MEJ, Rabelink TJ, Schaapherder AFM. Donor brain death predisposes human kidney grafts to a proinflammatory reaction after transplantation. Am J Transplant 2011; 11:1064-70.

10 Kuecuek O, Mantouvalou L, Klemz R et al. Significant reduction of proinflammatory cytokines by treatment of the brain-dead donor. Transplant Proc 2005; 37:387-8.

11 Aiello S, Cassis P, Mister M et al. Rabbit anti-rat thymocyte immunoglobulin preserves renal function during ischemia/reperfusion injury in rat kidney. Transpl Int 2011; 24:829-38.

12 Zhou H, Liu J, Pan P, Jin D, Ding W, Li W. Carbon monoxide inhalation decreased lung injury via anti-inflammatory and antiapoptotic effects in brain death rats. Exp Biol Med (Maywood) 2010; 235:1236-43.

13 Conductier G, Blondeau N, Guyon A, Nahon JL, Rovère C. The role of monocyte chemoattractant protein MCP1/CCL2 in neuroinflammatory diseases. J Neuroimmunol 2010; 224:93-100.

14 Singbartl K, Ley K. Protection from ischemia-reperfusion induced severe acute renal failure by blocking E-selectin. Crit Care Med 2000; 28:2507-14.

15 Singbartl K, Green SA, Ley K. Blocking P-selectin protects from ischemia/reperfusion-induced acute renal failure. FASEB J 2000; 14:48-54.

16 Damman J, Hoeger S, Boneschansker L et al. Targeting complement activation in brain-dead donors improves renal function after transplantation. Transplant Immunol 2011; 24:233-7.

17 Beiras-Fernandez A, Walther S, Thein E, Muenzing S, Hammer C. Influence of polyclonal ATGs on expression of adhesion molecules: an experimental study. Transplant Proc 2005; 37:1944-6.

18 Preville X, Flacher M, LeMauff B et al. Mechanisms involved in antithymocyte globulin immunosuppressive activity in a nonhuman primate model. Transplantation 2001; 71:460-8.

19 Donnahoo KK, Shames BD, Harken AH, Meldrum DR. Review article: the role of tumor necrosis factor in renal ischemiareperfusion injury. J Urol 1999; 162:196-203.

20 Farrar CA, Zhou W, Lin T, Sacks SH. Local extravascular pool of C3 is a determinant of postischemic acute renal failure. FASEB J 2006; 20:217-26.

21 Guttman RD, Caudrelier P. Pharmacokinetics, foreign protein immune response, cytokine release and lymphocyte subsets in patients receiving thymoglobuline and immunosuppression. Transplant Proc 1997; 29:24-6S.

22 Pratt JR, Basheer SA, Sacks SH. Local synthesis of complement component $\mathrm{C} 3$ regulates acute renal transplant rejection. Nat Med 2002; 8:582-7.

23 Naesens M, Li L, Ying L et al. Expression of complement components differs between kidney allografts from living and deceased donors. J Am Soc Nephrol 2009; 20:1839-51.

24 Zeller JC, Panoskaltsis-Mortari A, Murphy WJ et al. Induction of CD4+ T cell alloantigen-specific hyporesponsiveness by IL-10 and TGF-beta. J Immunol 1999; 163:3684-91.

25 Lopez M, Clarkson MR, Albin M, Sayegh MH, Najafian N. A novel mechanism of action for anti-thymocyte globulin: induction of 
CD4+CD25+Foxp3+ regulatory T cells. J Am Soc Nephrol 2006; 17:2844-53.

26 De Serres SA, Yeung MY, Mfarrej BG, Najafian N. Effect of biologic agents on regulatory T cells. Transplant Rev (Orlando) 2011; 25:110-16.

27 Liu Z, Fang Y, Wang X et al. Upregulation of molecules associated with T-regulatory function by thymoglobulin pretreatment of human CD4+ cells. Transplantation 2008; 86:1419-26.
28 Boenisch O, Lopez M, Elyaman W, Magee CN, Ahmad U, Najafian N. Ex vivo expansion of human Tregs by rabbit ATG is dependent on intact STAT3-signaling in CD4+ T cells and requires the presence of monocytes. Am J Transplant 2012; 12:856-66; doi: 10.1111/ j.1600-6143.2011.03978.x.

29 Kolattukudy PE, Quach T, Bergese S et al. Myocarditis induced by targeted expression of the MCP-1 gene in murine cardiac muscle. Am J Pathol 1998; 152:101-11. 